\title{
Disease activity score-driven therapy versus routine care in patients with recent-onset active rheumatoid arthritis: data from the GUEPARD trial and ESPOIR cohort
}

\author{
M Soubrier, ${ }^{1}$ C Lukas, ${ }^{2}$ J Sibilia, ${ }^{3}$ B Fautrel, ${ }^{4}$ F Roux,${ }^{5}$ L Gossec, ${ }^{6}$ S Patternotte, ${ }^{6}$ \\ M Dougados $^{6}$
}

${ }^{1}$ Department of Rheumatology, Hôpital G Montpied, ClermontFerrand, France

2Department of Rheumatology, Hôpital Lapeyronie, Montpellier France

${ }^{3}$ Department of Rheumatology, Hôpital Hautepierre, Strasbourg, France

${ }^{4}$ Department of Rheumatology, Hôpital la Pitié Salpétrière, Paris, France

${ }^{5}$ Department of Biostatistics, RCTs, Lyon, France

${ }^{6}$ Department of Rheumatology B, Hôpital Cochin, Paris, France

\section{Correspondence to}

Dr M Soubrier, Department of Rheumatology, Hôpital G Montpied, 63003 ClermontFerrand, France:

msoubrier@

chu-clermontferrand.fr

Accepted 7 November 2010 Published Online First

17 January 2011

\begin{abstract}
Objectives To compare the efficacy of disease activity score in 28 joints (DAS28ESR)-driven therapy with antitumour necrosis factor (patients from the GUEPARD trial) and routine care in patients with recent-onset rheumatoid arthritis (patients of the ESPOIR cohort).

Results After matching GUEPARD and ESPOIR patients on the basis of a propensity score and a 1:2 ratio, at baseline all patients had comparable demographic characteristics, rheumatoid factor, anticyclic citrullinated peptide antibody positivity and clinical disease activity parameters: erythrocyte sedimentation rate, C-reactive protein, mean DAS (6.26 \pm 0.87$)$, Sharp/van der Heijde radiographic score (SHS), health assessment questionnaire (HAQ). Disease duration was longer in GUEPARD patients $(5.6 \pm 4.6$ vs $3.5 \pm 2.0$ months, $p<0.001)$. After 1 year, the percentage of patients in remission with an $\mathrm{HAO}(<0.5)$ and an absence of radiological progression was higher in the tight control group $(32.3 \%$ vs $10.2 \%, p=0.011)$ as well as the percentage of patients in low DAS with an $\mathrm{HAO}(<0.5)$ and an absence of radiological progression $(36.1 \%$ vs $18.9 \%, p=0.045)$. However, there was no difference in the decrease in DAS, nor in the percentage of EULAR (good and moderate), ACR20, ACR50 and ACR70 responses. More patients in the tight control group had an $\mathrm{HAO}$ below 0.5 (70.2\% vs $45.2 \%, p=0.005)$. Overall, pain, patient and physician assessment and fatigue decreased more in the tight control group. The mean SHS progression was similar in the two groups as was the percentage of patients without progression.

Conclusions In patients with recent onset active rheumatoid arthritis, a tight control of disease activity allows more patients to achieve remission without disability and radiographic progression.
\end{abstract}

The management of early rheumatoid arthritis (RA) has evolved rapidly over the past decade as emphasised in the last EULAR recommendations for the management of RA. ${ }^{1}$ Synthetic diseasemodifying antirheumatic drugs (DMARD) should be started as soon as the diagnosis of RA is made and methotrexate is considered as the anchor drug. ${ }^{1}$ Biological agents, particularly tumour necrosis factor (TNF) inhibitor, have proved to be more effective than methotrexate as treatment for patients with newly diagnosed RA. ${ }^{2}$ Finally, patients with early active disease may benefit from an active steering and prompt adjustment of treatment intensity. ${ }^{3}$ GUEPARD is a prospective unblinded randomised multicentre controlled 1-year trial comparing two initial treatment strategies (initial methotrexate monotherapy vs its combination with adalimumab) in patients with early and active RA (<6 months, DAS28 $>5.1) .{ }^{4}$ In both groups, treatment was adjusted every 3 months with the aim of achieving a low DAS (DAS28ESR $<3.2)$ by the use of TNF blockers. We hypothesised that the systemic measurement of disease activity with adjustments to therapy according to a fixed protocol based mainly on anti-TNF results in a better clinical improvement and radiographic outcome than the routine care administered to patients of the ESPOIR cohort. ${ }^{5}$

\section{MATERIALS AND METHODS Patients}

In the GUEPARD study patients with early RA, as defined by the 1987 criteria of the American College of Rheumatology (ACR; formerly the American Rheumatism Association), were recruited between May 2004 and May 2006 in 13 centres in France. Patients had a maximum disease duration of 6 months, were at least 18 years of age, and had active disease defined as a disease activity score in 28 joints (DAS28ESR) greater than 5.1. They were randomly assigned to receive methotrexate monotherapy (group 1, $\mathrm{n}=32$; methotrexate $0.3 \mathrm{mg} / \mathrm{kg}$ per week, maximum of $20 \mathrm{mg} / \mathrm{kg}$ per week, without escalating dose regimen) or initial combination therapy (group 2, n=33) with methotrexate (same regimen as group 1) and adalimumab (40 mg every otherweek). In both groups, treatment was adjusted every 3 months. The aim was to achieve a low disease activity state (DAS28ESR <3.2). For example, in the event of persistent active disease at month 3 , adalimumab was added (group 1) or increased to $40 \mathrm{mg} /$ week (group 2). Three months later, in the event of a low disease activity state, methotrexate was continued for a further 3 months and thereafter the dose was slowly tapered (group1) or adalimumab was decreased for 3 months and then discontinued at month 9 and thereafter restarted in the event of flare (group 2). ${ }^{4}$

The ESPOIR cohort is a nationwide, longitudinal, prospective cohort of 813 patients established by the French Society for Rheumatology to investigate the diagnosis, outcome markers, epidemiology, pathogenesis and medico-economics of early arthritis and RA. ${ }^{5}$ The cohort was constituted by online under the BMJ Journals unlocked scheme, see http:// ard.bmj.com/info/unlocked.dtl 
having general practitioners and rheumatologists refer patients with early arthritis to hospitals participating in the ESPOIR cohort project. Patients were eligible for inclusion in the cohort if they had a definitive or probable clinical diagnosis of RA or a diagnosis of undifferentiated arthritis with potential for progressing to RA. Patients were included if they met the following criteria: age over 18 years and less than 70 years, swelling of two or more joints for 6 weeks, symptom duration of less than 6 months and no previous treatment with DMARD or glucocorticoids. However, the use of glucocorticoids for no longer than 2 weeks with a mean dosage of no greater than $20 \mathrm{mg}$ per day and discontinuation at least 2 weeks earlier did not prevent study inclusion. Patients who were included in the cohort were assessed every 6 months for 2 years and then once a year for at least 10 years.

\section{Assessment of variables}

In the GUEPARD study and in the ESPOIR cohort the following variables were assessed: number of swollen joints (0-28); number of tender joints (0-28); visual analogue scale (VAS) score for pain (0-100 mm); VAS general wellbeing (0-100); VAS fatigue (0-100); VAS physician overall assessment (0-100); morning stiffness (min); erythrocyte sedimentation rate (ESR, $\mathrm{mm} / \mathrm{h}^{1 \mathrm{st}}$ ) and C-reactive protein (CRP, $\left.\mathrm{mg} / \mathrm{l}\right)$. These variables were assessed monthly in the GUEPARD study, and at weeks 0, 24 and 52 in the ESPOIR cohort. The French version of the health assessment questionnaire (HAQ) was filled out at weeks $0,12,24,36$ and 52 in the GUEPARD study and at weeks 0 , 24 and 52 in the ESPOIR cohort. (0-3=greatest functional disability). Rheumatoid factor (RF) and anticyclic citrullinated peptide antibody (anti-CCP) status was defined at baseline. Radiographs of hands and feet were taken at baseline, at week 24 and at week 52 in the GUEPARD study and at baseline and week 52 in the ESPOIR cohort. Radiographs were independently scored by a trained assessor (CL), who was blinded to the patient's identity and treatment centre according to the modified Sharp/van der Heijde score (SHS), with a range of 0 to 448. A patient was classified as having erosive disease if the erosion score was greater than 1 .

\section{Matching methodology}

Patients included in the ESPOIR cohort who fulfilled the inclusion criteria of the GUEPARD trial (DAS $\geq 5.1$, ACR 1987 criteria fulfilled) without any participation in a study protocol and no missing baseline data were eligible $(n=277)$ for the matching procedure. In order to reduce bias in baseline measured characteristics, patients were then matched using the propensity score, ie, the predicted probability of a patient belonging to the tight control study. The propensity score was computed using a multivariate logistic regression model. All demographic and disease characteristics at baseline were used as covariates in the model: centre, gender, age at inclusion, disease duration, body mass index, RF and anti-CCP status, ESR, CRP, tender and swollen joint count (28), patient's assessments of pain, disease activity, fatigue and doctor's assessment of disease activity on a 0-100 mm VAS, HAQ, physical and mental components of SF36, erosive disease, erosion score and narrowing score. The propensity score was reliable because the overall model fit probability was less than $10^{-4}$ and the area under the receiver operating characteristic curve (95\% CI) was 0.76 (0.71 to 0.83$)$.

The matching procedure used was an iterative process based on the least propensity score difference between case and control. ${ }^{6}$ The rule used to determine the number of controls to match each case was the highest statistical power with the best goodness of fit of matched pairs. The statistical power for a 1:1 matching was too low (71\%) in comparison with a 1:2 matching $(83 \%)$, assuming a comparison of two binomial proportions of $40 \%$ ( $n=65$ ) versus $20 \%$ ( $n=65$ or 130 ). Moreover, when the matching procedure was applied to a 1:3 matching, the goodness of matched pairs was lower than the 1:2 matching. Consequently, the 1:2 matching was computed.

\section{Statistical analysis}

All matched patients ( $\mathrm{n}=195)$ were included in an intent-tostudy analysis of efficacy and safety. For radiographic criteria, missing evaluations at month $12(\mathrm{~N}=10 / 195)$ were replaced by linear regression imputation using baseline and month 6 evaluations. For all other criteria, missing data (from $2.7 \%$ to $4.8 \%$ ) were replaced using linear regression between previous and next values, if available. Otherwise, last observation carried forward methodology was used. At baseline, comparability between groups was assessed using Student's t tests or Fisher's exact tests. After 6 months and 1 year, both groups were compared using analyses of covariance for continuous data or logistic regression models for discrete data. The strategy group (tight control vs routine practice), the mean daily dosage of corticosteroids and the duration of biotherapy during the study were used as covariates. $p$ Values less than 0.05 were considered statistically significant.

\section{RESULTS}

At baseline, all patients in the tight control group $(n=65)$ and routine practice group $(n=130)$ fulfilled the ACR 1987 criteria. They had comparable demographic characteristics (female $77 \%$, age $48.0 \pm 13.0$ years), RF and anti-CCP positivity $(67.2 \%$ and $63.6 \%$ ), swollen and tender joint counts (10.5 \pm 5.5 and $14.0 \pm 6.8)$, patient's assessment of global disease activity $(68.0 \pm 20.8 \mathrm{~mm})$, pain $(57.0 \pm 21.2 \mathrm{~mm})$, fatigue $(54.7 \pm 26.3 \mathrm{mg})$, doctor's assessment $(66.5 \pm 17.8 \mathrm{mg})$ of disease activity on a 0-100 mg VAS, ESR (38.7 \pm 24.6 mg first hour), CRP $(29.4 \pm 32.6$ $\mathrm{mg} / \mathrm{l})$, typical erosive disease $(17.9 \%)$, modified total SHS $(6.5 \pm 10.1)$, erosion score $(2.4 \pm 4.2)$, narrowing score $(4.1 \pm 7.1)$, $\mathrm{HAQ}(1.3 \pm 0.6)$, physical (35.5 \pm 7.9$)$ and mental components (37.5 \pm 11.5$)$ of SF36. All patients had a DAS score above 5.1 (mean 6.26 \pm 0.87 ). The tight control group had a longer mean

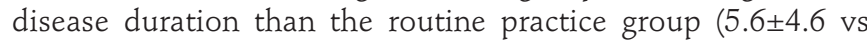
$3.5 \pm 2.0$ months, $p<0.001$; table 1$)$. All patients in the GUEPARD trial but only $74.8 \%$ in the ESPOIR cohort received methotrexate $(p<0.0001)$. Other patients in ESPOIR cohort received sulphasalazine $(9.2 \%)$, leflunomide $(5.2 \%)$ or hydroxychloroquine $(7.7 \%)$. None received combination DMARD therapy. More patients in the GUEPARD trial received anti-TNF than in the ESPOIR cohort ( $89 \%$ vs $13.08 \%, \mathrm{p}<0.0001)$. In contrast, more patients in the ESPOIR cohort received corticosteroids $(67 \%$ vs $43 \%, p=0.002)$ with a greater cumulative dose $(3316 \pm 1842$ vs $1808 \pm 1110, \mathrm{p}<0.0001)$ and mean dose $(8.66 \pm 8.51 \mathrm{mg}$ vs $4.86 \pm 3.27 \mathrm{mg}, \mathrm{p}=0.0009)$. Thirteen patients received intraarticular steroid injections in the GUEPARD study and 38 in the ESPOIR cohort $(p=0.2)$.

After 1 year, the percentage of patients in remission with an $\mathrm{HAQ}(<0.5)$ and an absence of radiological progression was higher in the tight control group $(32.3 \%$ vs $10.2 \%, p=0.011)$ as well as the percentage of patients in low DAS with an HAQ $(<0.5)$ and an absence of radiological progression $(36.1 \%$ vs $18.9 \%, p=0.045$; figure 1). However, there was no difference in the decrease in DAS (tight control: $-3.12 \pm 1.82$; routine practice: $-2.65 \pm 1.66, p=0.12)$, nor in the percentage of patients in low 
Table 1 Baseline characteristics

\begin{tabular}{|c|c|c|c|}
\hline & $\begin{array}{l}\text { GUEPARD } \\
(\mathrm{N}=65)\end{array}$ & $\begin{array}{l}\text { ESPOIR } \\
(N=130)\end{array}$ & p Value \\
\hline Age mean $\pm S D$ (years) & $47.9 \pm 15.7$ & $48.1 \pm 11.5$ & 0.92 \\
\hline Women, n (\%) & $52(80.0)$ & $98(75.4)$ & 0.59 \\
\hline BMI $\left(\mathrm{kg} / \mathrm{m}^{2}\right)$ & $26.0 \pm 4.7$ & $26.4 \pm 5.5$ & 0.59 \\
\hline Symptom duration, months, mean $\pm S D$ & $5.6 \pm 4.6$ & $3.5 \pm 2.0$ & $<0.001$ \\
\hline IgM rheumatoid factor positive, n (\%) & $46(70.8)$ & $85(65.4)$ & 0.52 \\
\hline Anti-CCP positive, n (\%) & $46(70.8)$ & $78(60.0)$ & 0.16 \\
\hline Pain (VAS 0-100) mean \pm SD & $59.5 \pm 22.0$ & $55.7 \pm 20.7$ & 0.23 \\
\hline $\begin{array}{l}\text { Patient global assessment (VAS 0-100) } \\
\text { mean } \pm \text { SD }\end{array}$ & $68.1 \pm 18.7$ & $67.9 \pm 21.9$ & 0.93 \\
\hline Fatigue (VAS 0-100) mean \pm SD & $57.4 \pm 23.6$ & $53.4 \pm 27.6$ & 0.31 \\
\hline $\begin{array}{l}\text { Physician global assessment (VAS 0-100) } \\
\text { mean } \pm \text { SD }\end{array}$ & $67.6 \pm 17.2$ & $65.9 \pm 18.1$ & 0.53 \\
\hline Tender joint count (mean \pm SD) & $14.0 \pm 6.7$ & $14.1 \pm 6.8$ & 0.93 \\
\hline Swollen joint count (mean \pm SD) & $10.1 \pm 4.9$ & $10.7 \pm 5.7$ & 0.51 \\
\hline ESR (mean $\pm S D)$ & $37.3 \pm 22.4$ & $39.5 \pm 25.7$ & 0.56 \\
\hline $\mathrm{CRP}(\mathrm{mg} / \mathrm{l})$ mean $\pm \mathrm{SD}$ & $28.7 \pm 33.4$ & $29.8 \pm 32.3$ & 0.83 \\
\hline DAS28ESR, mean $\pm S D$ & $6.24 \pm 0.81$ & $6.27 \pm 0.90$ & 0.84 \\
\hline DAS28CRP, mean \pm SD & $5.82 \pm 0.86$ & $5.87 \pm 0.95$ & 0.72 \\
\hline HAQ score, 0-3 scale, mean \pm SD & $1.37 \pm 0.65$ & $1.28 \pm 0.67$ & 0.39 \\
\hline Physical component summaries of SF-36 & $35.4 \pm 8.3$ & $35.6 \pm 7.8$ & 0.86 \\
\hline Mental component summaries of SF-36 & $37.7 \pm 11.9$ & $37.5 \pm 11.3$ & 0.87 \\
\hline Total SHS, 0-448 scale, mean \pm SD & $7.7 \pm 13.4$ & $5.8 \pm 8.0$ & 0.30 \\
\hline Erosion score, 0-280 scale, mean \pm SD & $2.4 \pm 4.9$ & $2.4 \pm 3.7$ & 0.97 \\
\hline JSN score, 0-168 scale, mean \pm SD & $5.3 \pm 9.5$ & $3.5 \pm 5.4$ & 0.15 \\
\hline Typical erosive disease, n (\%) & $13(20.0)$ & $22(16.9)$ & 0.69 \\
\hline
\end{tabular}

$\mathrm{BMI}$, body mass index; CRP, C-reactive protein; DAS28, disease activity score in 28 joints; ESR, erythrocyte sedimentation rate; $\mathrm{HAO}$, health assessment questionnaire; JSN, joint-space narrowing; SHS, modified Sharp/van der Heijde score; SF-36, short form 36 health survey; VAS, visual analogue scale.
DAS (tight control: $63.1 \%$; routine practice: $43.8 \%, \mathrm{p}=0.64$ ) and in remission (tight control: $47.7 \%$; routine practice: $29.2 \%$, $\mathrm{p}=0.35$; table 2 and figure 1 ). There was no statistically significant difference in EULAR (good and moderate), ACR20, ACR50, and ACR70 responses (93.8\% vs $84.6 \%, p=0.11 ; 81.5 \%$ vs $64 \%, p=0.37 ; 68.8 \%$ vs $41.3 \%, p=0.43 ; 50.8 \%$ vs $23.6 \%$, $\mathrm{p}=0.07$; figure 1). The improvement in $\mathrm{HAQ}$ was similar (tight control: $-0.94 \pm 0.82$; routine practice: $-0.68 \pm 0.73, p=0.07$ ) but more patients in the tight control group had an HAQ below 0.5 (70.2\% vs $45.2 \%, \mathrm{p}=0.005)$. Overall, pain, patient and physician assessment and fatigue decreased more in the tight control group than in the routine practice group $(-44.8 \pm 31.9$ vs $-24.2 \pm 29.2$, $\mathrm{p}<0.001 ;-46.6 \pm 35.6$ vs $-32.3 \pm 32.5, \mathrm{p}=0.015 ;-49.1 \pm 32.7$ vs $36.0 \pm 29.9, p=0.02 ;-37.1 \pm 36.2$ vs $-12.2 \pm 33.1, p<0.0001)$. The mean SHS progression was similar in the two groups (tight control: $0.95 \pm 4.6$; routine practice: $1.5 \pm 4.1, \mathrm{p}=0.46$ ) as was the percentage of patients without progression $(71.19 \%$ vs $69.23 \%$, $\mathrm{p}=0.37$; table 2). In the GUEPARD trial, malignancy was diagnosed in two patients (ovarian carcinoma, pancreatic cancer) both of whom were in the methotrexate and adalimumab group. No patients had serious infections. The other serious adverse events in the GUEPARD trial attributed to the treatment were hepatitis in two patients, methotrexate pneumonia in one patient and weight loss in another patient. Seven adverse events were observed in the ESPOIR cohort. Two patients had dyspnoea related to methotrexate, two had haematemesis, one had soft tissue infection (patient on methotrexate), one had thrombopaenia related to methotrexate and one had pericarditis related to salazopyrin.

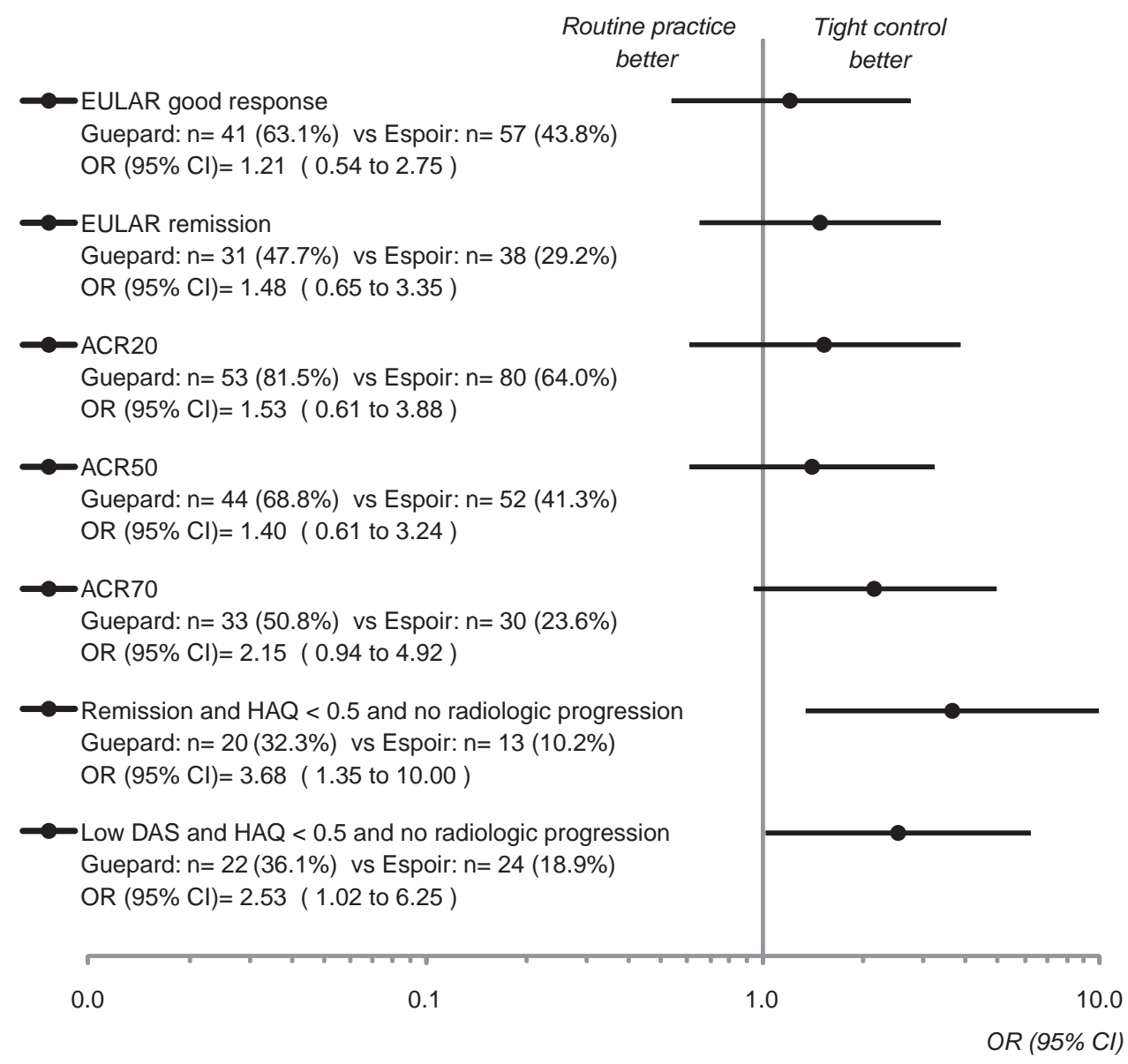

Figure 1 Patients in whom response had been achieved at the 12 month assessment. ACR, Americal College of Rheumatology; DAS, disease activity score; $\mathrm{HAO}$, health assessment questionnaire. 
Table 2 Change in disease activity, radiographic damage, physical function and quality of life between baseline and 12 months

\begin{tabular}{|c|c|c|c|}
\hline & GUEPARD ( $\mathrm{N}=65$ ) & ESPOIR (N=130) & p Value \\
\hline DAS28 & $-3.12(-3.56$ to -2.67$)$ & $-2.65(-2.94$ to -2.37$)$ & 0.12 \\
\hline Tender joint count (range 0-28) & $-10.1(-12.4$ to -7.9$)$ & $-9.1(-10.6$ to -7.6$)$ & 0.51 \\
\hline Swollen joint count (range 0-28) & $-7.7(-9.5$ to -5.9$)$ & $-8.0(-9.1$ to -6.8$)$ & 0.84 \\
\hline Patient global assessment (range 0-100 mm VAS) & $-46.6(-55.3$ to -37.9$)$ & $-32.3(-37.9$ to -26.7$)$ & 0.02 \\
\hline Physician global assessment (range 0-100 mm VAS) & $-49.1(-57.1$ to -41.1$)$ & $-36.0(-41.1$ to -30.8$)$ & 0.02 \\
\hline $\mathrm{ESR}, \mathrm{mm} / \mathrm{h}$ & $-19.5(-26.7$ to -12.3$)$ & $-20.6(-25.3$ to -16.0$)$ & 0.82 \\
\hline Fatigue (range $0-100 \mathrm{~mm}$ VAS) & $-37.1(-46.0$ to -28.3$)$ & $-12.2(-17.9$ to -6.5$)$ & $<0.0001$ \\
\hline Physical component summaries of SF-36 (range $0-100$ ) & 9.66 (6.73 to 12.59$)$ & 8.75 (6.97 to 10.53$)$ & 0.64 \\
\hline Mental component summaries of SF-36 (range $0-100$ ) & 9.26 (5.38 to 13.14$)$ & $3.92(1.56$ to 6.27$)$ & 0.04 \\
\hline Erosion score, $0-280$ scale & $0.4(-0.6$ to 1.3$)$ & $1.3(0.8$ to 1.9$)$ & 0.12 \\
\hline JSN score, 0-168 scale & $0.6(0.2$ to 1.0$)$ & $0.2(0.0$ to 0.4$)$ & 0.13 \\
\hline Total SHS, 0-448 scale & $0.9(-0.2$ to 2.1$)$ & 1.5 (0.8 to 2.3$)$ & 0.46 \\
\hline
\end{tabular}

Mean change $(95 \% \mathrm{Cl})$.

CRP, C-reactive protein; DAS28, disease activity score in 28 joints; ESR, erythrocyte sedimentation rate; HAQ, health assessment questionnaire; JSN, joint-space narrowing; SHS modified Sharp/van der Heijde score; VAS, visual analogue scale.

\section{DISCUSSION}

In patients with recent-onset active RA, a tight control of disease activity with TNF blockers, as implemented in the GUEPARD trial, allowed more patients to achieve remission without disability and radiographic progression than the routine care administered to the patients in the ESPOIR cohort. In addition, patients with tight control had better improvements in $\mathrm{HAO}$, pain, fatigue and global assessment of disease activity, suggesting a Hawthorne effect. ${ }^{7}$ However, the decrease in DAS, the number of patients in low DAS or in remission and radiographic progression were similar in the two groups. These results conflict with those previously observed with conventional DMARD. In the TICORA study the decrease in DAS was greater in the intensive group ( $-3.5 \mathrm{vs}-1.9$, $\mathrm{p}<0.0001)$, as was the number of patients in remission $(65 \%$ vs $16 \%, \mathrm{p}<00001) .{ }^{8}$ Patients in the intensive group had a reduced progression of erosion score and total Sharp scores, but no difference was noted in the progression of joint-space narrowing. However, the patients assigned to the intensive group were seen every month and any swollen joint was injected unless the joint had been injected during the previous 3 months or if the patients declined. Furthermore, in the first 3 months of starting a new DMARD if $120 \mathrm{mg}$ of triamcilone acetonide was not injected intra-articularly, the same dose was given intramuscularly if the disease activity score remained more than 2,4. In the CAMERA study, all clinical variables (tender and swollen joint counts, pain, general wellbeing) improved significantly in the first year in the intensive strategy group in comparison with the conventional strategy group, with the exception, however, of ESR, morning stiffness and functional disability. ${ }^{9}$ The results of radiographic progression were unavailable at 1 year. More recently, the results of patients receiving sequential monotherapy or step up to combination therapy in the BeSt trial were compared with the results of similar RA patients who had received routine care. ${ }^{10}$ Again, patients with DAS-driven therapy despite higher baseline scores had better clinical outcomes after 1 year of follow-up. The mean HAQ improvement was 0.70 versus $0.55(p=0.029)$, the mean DAS28 improvement was 2.7 versus $1.9(\mathrm{p}<0.001)$ and the median ESR improvement was $19 \mathrm{mg} / \mathrm{h}$ versus $13 \mathrm{~mm} / \mathrm{h}(\mathrm{p}=0.011)$. The percentage of patients in clinical remission (DAS28 <2.6) after 1 year was $31 \%$ versus $18 \%(p=0.005)$. In the DAS-driven therapy group, median SHS progression was 2.0 (expected progression 7.0), and in routine care patients it was 1.0 (expected progression 4.4). The larger difference between the expected progression and observed progression in the DAS-driven group suggests that the suppression of joint damage progression was better in this group than in routine care patients, albeit not to a level of significance $(p=0.126) .{ }^{10}$ The lack of difference in our study could be explained by the fact that the investigators of the ESPOIR cohort were aware of early RA. ${ }^{11}$ Therefore, in the ESPOIR cohort, baseline tender joint count (>9), abnormal CRP levels and anti-CCP antibodies were closely related to DMARD treatment ${ }^{11}$ In addition, it is likely that the publication of the preliminary results from the TICORA study in 2003, by underlining the interest of tight control, had influenced the therapeutic care of patients of the ESPOIR cohort. $^{12}$ Nevertheless, a tight control of disease activity with TNF blockers allows more patients to achieve remission without disability and radiographic progression.

Acknowledgements The authors wish to thank all the investigators who recruited and followed the patients (F Beranbaum, Paris-Saint Antoine; MC Boissier, ParisBobigny; A Cantagrel, Toulouse; B Combe, Montpellier; M Dougados, Paris-Cochin; P Fardelonne, Amiens; B Fautrel, P Bourgeois, Paris-La Pitié; RM Flipo, Lille; P Goupille, Tours; F Liote, Paris-Lariboisière; X Le Loet, Rouen; X Mariette, Paris-Bicetre; 0 Meyer, Paris-Bichat; A Saraux, Brest; T Schaeverbeke, Bordeaux; J Sibilia, Strasbourg) and Catherine Le Bourlout, clinical research nurse (Cochin, Paris).

Competing interests None.

Funding This work was supported by an unrestricted grant of Abbott. An unrestricted grant from Merck Sharp and Dohme was allocated for the first 5 years of the ESPOIR cohort. Two additional grants from INSERM were obtained to support part of the biological database. The French Society of Rheumatology, Abbott, Amgen and Wyeth also supported the ESPOIR cohort study.

Patient consent Obtained.

Ethics approval This study was conducted with the approval of the ESPOIR central ethics committee of Montpellier and the GUEPARD central ethics committee of Cochin, Paris.

Provenance and peer review Not commissioned; externally peer reviewed.

\section{REFERENCES}

1. Smolen JS, Landewé R, Breedveld FC, et al. EULAR recommendations for the management of rheumatoid arthritis with synthetic and biological disease-modifying antirheumatic drugs. Ann Rheum Dis 2010;69:964-75.

2. Nam JL, Winthrop KL, van Vollenhoven RF, et al. Current evidence for the management of rheumatoid arthritis with biological disease-modifying antirheumatic drugs: a systematic literature review informing the EULAR recommendations for the management of RA. Ann Rheum Dis 2010;69:976-86.

3. Knevel R, Schoels M, Huizinga TW, et al. Current evidence for a strategic approach to the management of rheumatoid arthritis with disease-modifying antirheumatic drugs: a systematic literature review informing the EULAR recommendations for the management of rheumatoid arthritis. Ann Rheum Dis 2010;69:987-94. 
4. Soubrier M, Puéchal X, Sibilia J, et al. Evaluation of two strategies (initial methotrexate monotherapy vs its combination with adalimumab) in management of early active rheumatoid arthritis: data from the GUEPARD trial. Rheumatology (Oxford) 2009; 48:1429-34

5. Combe B, Benessiano J, Berenbaum F, et al. The ESPOIR cohort: a ten-year follow-up of early arthritis in France: methodology and baseline characteristics of the 813 included patients. Joint Bone Spine 2007;74:440-5.

6. Parson LS. Performing a 1:N case-control match on propensity score. Proceeding of the 29th Annual SAS Users Group International Conference. SAS Institute, Montreal, Canada, 9-12 May 2004

7. Wolfe $\mathbf{F}$, Michaud K. The Hawthorne effect, sponsored trials, and the overestimation of treatment effectiveness. J Rheumatol 2010;37:2216-20.

8. Grigor C, Capell H, Stirling A, et al. Effect of a treatment strategy of tight control for rheumatoid arthritis (the TICORA study): a single-blind randomised controlled trial. Lancet 2004;364:263-9.
9. Verstappen SM, Jacobs JW, van der Veen MJ, et al. Intensive treatment with methotrexate in early rheumatoid arthritis: aiming for remission. Computer Assisted Management in Early Rheumatoid Arthritis (CAMERA, an open-label strategy trial). Ann Rheum Dis 2007;66:1443-9.

10. Goekoop-Ruiterman YP, de Vries-Bouwstra JK, Kerstens PJ, et al. DAS-driven therapy versus routine care in patients with recent-onset active rheumatoid arthritis. Ann Rheum Dis 2010;69:65-9.

11. Lukas C, Guillemin F, Landewé $\mathrm{R}$, et al. Factors determining a DMARD initiation in early inflammatory arthritis patients. The ESPOIR cohort study. Clin Exp Rheumato 2009:27:84-91.

12. Porter DR, Grigor C, Stirling A, et al. A randomised controlled trial of a strategy of tight control of disease activity in rheumatoid arthritis - outcome over 18 months. Arthritis Rheum 2003;48(Suppl):515. 